\title{
FAS system deregulation in T-cell lymphoblastic lymphoma
}

\author{
M Villa-Morales ${ }^{1,2,3}$, MA Cobos ${ }^{1,2,3}$, E González-Gugel ${ }^{4}$, V Álvarez-Iglesias ${ }^{5}$, B Martínez ${ }^{2,6}$, MA Piris ${ }^{7}$, A Carracedo ${ }^{5,8}$, J Benítez ${ }^{2,9}$ \\ and J Fernández-Piqueras ${ }^{\star, 1,2,3}$
}

The acquisition of resistance towards FAS-mediated apoptosis may be required for tumor formation. Tumors from various histological origins exhibit FAS mutations, the most frequent being hematological malignancies. However, data regarding FAS mutations or FAS signaling alterations are still lacking in precursor T-cell lymphoblastic lymphomas (T-LBLs). The available data on acute lymphoblastic leukemia, of precursor origin as well, indicate a low frequency of FAS mutations but often report a serious reduction in FAS-mediated apoptosis as well as chemoresistance, thus suggesting the occurrence of mechanisms able to deregulate the FAS signaling pathway, different from FAS mutation. Our aim at this study was to determine whether FAS-mediated apoptotic signaling is compromised in human T-LBL samples and the mechanisms involved. This study on 26 T-LBL samples confirms that the FAS system is impaired to a wide extent in these tumors, with $57.7 \%$ of the cases presenting any alteration of the pathway. A variety of mechanisms seems to be involved in such alteration, in order of frequency the downregulation of FAS, the deregulation of other members of the pathway and the occurrence of mutations at FAS. Considering these results together, it seems plausible to think of a cumulative effect of several alterations in each T-LBL, which in turn may result in FAS/FASLG system deregulation. Since defective FAS signaling may render the T-LBL tumor cells resistant to apoptotic cell death, the correct prognosis, diagnosis and thus the success of anticancer therapy may require such an in-depth knowledge of the complete scenario of FAS-signaling alterations.

Cell Death and Disease (2014) 5, e1110; doi:10.1038/cddis.2014.83; published online 6 March 2014

Subject Category: Cancer

Precursor T-cell lymphoblastic lymphomas (T-LBLs) are neoplasms derived from immature thymocytes at varying stages of differentiation. ${ }^{1}$ T-LBLs are aggressive malignancies that account for about one-third of childhood non-Hodgkin lymphomas (NHL). ${ }^{2}$ Small to medium-sized blast cells with round to irregular or convoluted nuclei and a high mitotic activity compose T-LBLs. In terms of immunophenotype, they are characteristically positive for terminal deoxynucleotidyl transferase $(\mathrm{TdT}){ }^{3}$ The molecular genetics of T-LBL are not well characterized, mainly due to the scarcity of samples for study.

FAS (CD95/APO-1) is a membrane receptor belonging to the tumor necrosis factor receptor (TNFR) superfamily whose mature canonical isoform (Ensemble FAS-005) encodes a type-1 transmembrane (TM) glycoprotein of $\sim 48 \mathrm{kDa} .{ }^{4}$ The ligand for FAS receptor FASLG (CD95L) belongs to the TNF superfamily. ${ }^{5}$ Its canonical isoform (Ensembl FASLG-001) encodes a type-2 TM protein of $40 \mathrm{kDa}$. Previously to the binding of the ligand and independently of this event, FAS molecules preassociate in homotrimers on the cell surface through their so-called pre-ligand assembly domain (PLAD). ${ }^{6}$ The TNF homology domain (THD) in FASLG mediates selfassociation into a compact ligand homotrimer that interacts with FAS through its cysteine-rich domains (CRDs). ${ }^{7}$ The ligand-activated receptor trimers recruit the adapter molecule FAS-associated death domain (FADD) by homotypic interaction through their death domains (DDs). FADD recruits the initiator procaspase 8 (CASP8) by the death effector domain (DED) to form the death-inducing signaling complex (DISC). ${ }^{8}$ Procaspase 8 is cleaved in the DISC to give rise to active caspase 8 , which dissociates from the DISC to proceed with the activation of the caspase cascade, ${ }^{9}$ eventually leading to apoptosis.

Peripheral T-cell homeostasis involves apoptosis mediated by the FAS system. Moreover, apoptosis during negative/ positive selection of thymocytes inside the thymus is certainly

\footnotetext{
${ }^{1}$ Centro de Biología Molecular Severo Ochoa, CSIC-UAM, Madrid, Spain; ${ }^{2}$ Centro de Investigación Biomédica en Red de Enfermedades Raras (CIBERER), Madrid, Spain; ${ }^{3}$ Instituto de Investigación Sanitario Fundación Jiménez Díaz, ISCIII, Madrid, Spain; ${ }^{4}$ Musculoskeletal Research Center, NYU Hospital for Joint Diseases, New York, NY, USA; ${ }^{5}$ Grupo de Medicina Xenómica, CIBERER, Universidade de Santiago de Compostela, Santiago de Compostela, Spain; ${ }^{6}$ Instituto de Investigación de Enfermedades Raras, ISCIII, Madrid, Spain; ${ }^{7}$ Hospital Universitario Marqués de Valdecilla, Fundación IFIMAV, Santander, Spain; ${ }^{8}$ Center of Excellence in Genomic Medicine Research, King Abdulaziz University, Jeddah, KSA and ${ }^{9}$ Human Genetics Group, CNIO, Madrid, Spain

*Corresponding author: J Fernández-Piqueras, Biología Celular e Inmunología, Centro de Biología Molecular Severo Ochoa (CBMSO), Universidad Autónoma de Madrid, Campus de Cantoblanco, C/Nicolás Cabrera 1, 28049 Madrid, Spain. Tel: +34 911964401; Fax: + 34 911964420; E-mail: jpiqueras@ cbm.uam.es Keywords: T-LBL; FAS-signaling; mutations; gene expression; apoptosis resistance

Abbreviations: ALL, acute lymphoblastic leukemia; BSA, bovine serum albumin; CRD, cysteine-rich domain; DD, death domain; DED, death effector domain; DISC, death-inducing signaling complex; DMEM, Dulbecco's modified Eagle's medium; DMSO, dimethyl sulfoxide; ECD, extracellular domain; FADD, FAS-associated death domain; ICD, intracellular domain; MEM, minimum essential medium; mFAS, membrane FAS; NHL, non-Hodgkin lymphomas; PI, propidium iodide; PLAD, pre-ligand assembly domain; qRT-PCR, quantitative reverse transcription-PCR; SFAS, soluble FAS; SP, signal peptide; TdT, terminal deoxynucleotidyl transferase; THD, TNF homology domain; T-LBL, T-cell lymphoblastic lymphoma; TM, transmembrane; TNFR, tumor necrosis factor receptor

Received 16.10.13; revised 17.1.14; accepted 04.2.14; Edited by T Brunner
} 
not only mediated by the FAS system, but it may participate in clonal deletion under certain circumstances. ${ }^{10}$ Undermining the immune system and suppressing the antitumor response are essential events during carcinogenesis allowing tumor formation. ${ }^{11}$ In this context, the FAS/FASLG system can elicit both tumorigenic and tumor-suppressing roles in the pathogenesis of cancer. ${ }^{11}$ However, FAS and FASLG genes have been traditionally considered tumor suppressor genes. ${ }^{12}$ This is supported by findings showing functional polymorphisms in Fas and Fas/ genes between strains of mice with extremely different susceptibilities to certain tumors. ${ }^{13-15}$

A causative relationship between the occurrence of $F A S$ mutations and cancer has been widely suggested in the literature. Tumors from various histological origins have been found to exhibit FAS mutations, the most frequent being, however, hematological malignancies. Interestingly, hot spots affecting exons 8 and 9 , mainly responsible for the intracellular domain (ICD) of FAS, have been described. Among them, some mutations are recurrent. ${ }^{16}$ However, data regarding FAS mutations or FAS signaling alterations are still lacking in T-LBL. The available data on acute lymphoblastic leukemia (ALL), of precursor origin as well, indicate a low frequency of FAS mutations but often report a serious reduction in FAS-mediated apoptosis as well as chemoresistance, ${ }^{17,18}$ thus suggesting the occurrence of mechanisms able to deregulate the FAS signaling pathway, different from FAS mutation.

Our aim at this study is to determine whether FAS-mediated apoptotic signaling is compromised in human T-LBL samples and the mechanism or mechanisms involved in such alteration. As various alterations affecting FAS signaling may confer resistance to malignant cells, a better knowledge of this scenario in T-LBL may become essential for prognosis, diagnosis and thus the success of therapy.

\section{Results}

Mutations affecting FAS in human T-LBL samples. FAS CDNAs from 26 human T-LBL samples were sequenced. We found that 5 out of the 26 samples (19.2\%) analyzed exhibited one or two heterozygous mutations at FAS. The mutations found were spread all along the FAS cDNA sequence (Figure 1), and none of them had been described previously in the literature. Among these five samples exhibiting FAS mutations, two of them (samples 00800SD1-1 and 04011685) had the same mutation, whereas the other three samples had different mutations each other (Tables 1 and 2). The mutations are defined according to the recommendations by the Human Genome Variation Society, last modified in 22 March 2013. ${ }^{19}$

In sample 000-220, a nucleotide substitution causes a missense mutation in exon 3 , which would affect the CRD2 domain. This sample exhibited a base insertion in exon 9 as well, which entailed a sequence frameshift responsible for the incorporation after residue 300 of a sequence of 19 amino acids not present in the wild-type sequence, together with the premature termination at codon 320 .

In samples 00800-SD1-1 and 04011685, the same base insertion in exon 4 caused sequence frameshift responsible for the substitution of cysteine in position 135 to leucine, together with the premature termination immediately at codon 136.

In sample 02M-121, a base substitution in exon 2 provoked a missense mutation at the signal peptide (SP). Interestingly, this sample showed as well the presence of an alternatively spliced isoform skipping exon 6, thus lacking the TM domain, which produces soluble FAS (sFAS) protein.

Sample 20040730 showed a base substitution at exon 4 causing a missense mutation, which affects CRD3.

The predicted damage of each mutation was determined using the Polyphen-2 software (biobyte solutions $\mathrm{GmbH}$, Heidelberg, Germany). On the basis of the mutation position, the software prediction and the literature, it has been predicted how each FAS mutant variant might behave in terms of FAS signaling (Table 1). Taken together, our results indicate that the occurrence of mutations in FAS might be involved, to a certain extent, in T-LBL in humans.

Mutant FAS variants present with altered in-vitro expression levels. We cloned each of the four different mutant FAS variants found among our T-LBL samples in pKEX vector and examined FAS protein expression after transfection of JK T cells (Figure 2a), using UB2 anti-FAS monoclonal antibody, known to target an identical epitope on the extracellular domain (ECD) of CD95 than $\mathrm{CH} 11$ anti-FAS antibody. ${ }^{20}$ Flow cytometry showed that JK cells have endogenous levels of membrane FAS (mFAS) of $34 \%$. Transient exogenous expression of wild-type FAS led to surface FAS levels of $70 \%$. In contrast, exogenous expression of 000-220, 00800-SD1-1 (identical to 04011685) and $02 \mathrm{M}-121$ mutant FAS variants showed mFAS levels of 47 , 38 and $29 \%$, respectively. Only the 20040730 mutant FAS variant exhibited a surface FAS level similar to that of the wild-type (64\%). These results suggest that the presence of the described mutations in FAS cDNA is sufficient for reduced mFAS expression in the cases of T-LBLs 000-220, 00800-SD1-1 and 02M-121, but not in the case of 20040730 (Figure 2a).

The 00800-SD1-1 variant predicted the synthesis of a peptide truncated before the TM domain. On the other side, the $02 \mathrm{M}-121$ variant predicted the synthesis of a peptide lacking the TM domain (sFAS). To determine whether these peptides were secreted, an adherent cell line with proved low endogenous expression of FAS, HEK-293T, was transfected with the wild-type FAS or each of the variants (Figure $2 b$ ), allowed for conditioning medium for $24 \mathrm{~h}$, and assessed for sFAS presence in cell extracts (panel 1) and extracellular conditioned medium (panel 2) through Western blot. In the case of the 02M-121 FAS variant, but not in the case of 00800SD1-1, sFAS was clearly detected. This result suggested that the 00800-SD1-1 mutant FAS might be degraded intracellularly.

Mutant FAS variant corresponding to case 000-220 exhibits defective FAS apoptotic signaling. To assess the functional impact of FAS mutations, each of the four different mutant $F A S$ variants were examined for FAS signal triggering following transfection of JK with our pKEX constructs (Figure $3 b-d$ ). FAS-mediated cell death was measured as propidium iodide $(\mathrm{PI})$ uptake after specific 
$\mathrm{CH} 11$ anti-FAS antibody treatment. $\mathrm{CH} 11$ antibody recognizes CRD2 and CRD3 regions of FAS and is known to recognize the same region of the death receptor than FASLG. ${ }^{21}$ Comparisons were made against wild-type FAS induction. Our results indicate that FAS signaling is impaired only in the case of 000-220, which may be due to reduced mFAS expression (as demonstrated previously, Figure 2), combined with inefficient DISC recruitment at its DD, as demonstrated by the impairment of caspase 8 activation (Figure 3d). Reduced mFAS expression shown in the cases of 00800-SD1-1 (or 04011685) and 02M-121 mutant FAS variants, or the production of SFAS in the latter (Figure 2), do not account for defective FAS signaling, however.

FAS expression levels are reduced in a significant fraction of human T-LBL samples. FAS expression was also analyzed in T-LBL samples. FAS mRNA expression level was determined using quantitative reverse transcription-PCR (qRT-PCR) when only RNA was available (Figure 4) and the FAS protein level was determined using western blot in those samples where protein extracts were available (Figure 5). Taken together, these results indicate

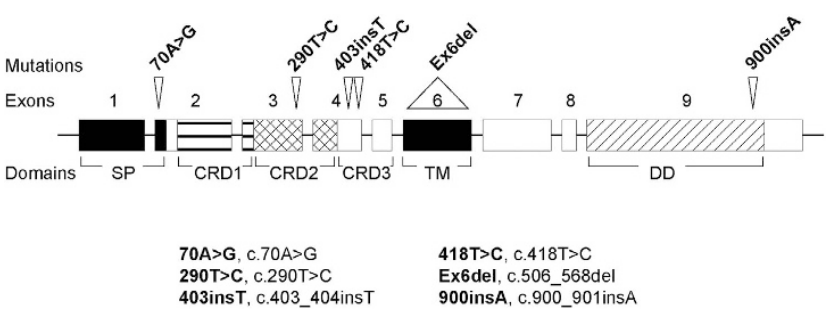

Figure 1 FAS mutations in T-LBL samples. Schematic representation of mutations found in FAS in human T-LBL samples, indicating FAS exons and FAS protein domains affected. An abbreviated form to indicate the mutations is included and explained in the legend that 10 out of the $26(38.5 \%)$ human T-LBL samples analyzed exhibited a clear reduction in the level of FAS expression (Table 2), compared with that of the JK cell line (proved to exhibit similar expression levels than immortalized human thymocytes, data not shown). Among the rest of them, FAS expression levels may vary, their reduction not being however so remarkable. Especially interesting is the fact that each of the human T-LBL samples already shown to bear mutations at FAS cDNA (000-220, 00800-SD1-1, 02M-121, 20040730 and 04011685) also exhibited reduction in FAS expression. Reduced FAS expression in 000-220, 00800-SD1-1, 02M-121 and 04011685 samples may be explained by the sole presence of their specific mutations, as already suggested by the in vitro expression of these mutant variants. In contrast, the striking reduction in FAS expression in 20040730 sample cannot be solely explained by the occurrence of a mutation in its FAS cDNA.

Taken together, our results from the tumor samples indicate that reduced FAS expression might be somehow contributing to T-LBL in humans. Moreover, this mechanism may exacerbate the effect of $F A S$ mutations, in terms of signaling alteration.

Altered status of the FAS signaling pathway in several human T-LBL samples. To further determine the status of the FAS signaling pathway in our human T-LBL samples, we assessed the protein levels of the main members of the pathway in the series of samples with available protein extracts. FASLG, FADD, CASP8 and CASP3 proteins were detected through western blot (Figure 5, Table 2).

Our results indicate that one sample (04010038) exhibited upregulated FASLG expression, whereas it was not detectable in the rest of the samples. FASLG upregulation in this sample, together with its self-protection through loss of FAS expression, might reflect one of the mechanisms involved in T-LBL occurrence in humans.

Table 1 Description of FAS mutations in T-LBL samples

\begin{tabular}{|c|c|c|c|c|c|}
\hline Sample & $\begin{array}{l}\text { Mutation in } \\
D N A^{\mathrm{a}}\end{array}$ & Change in protein & $\begin{array}{l}\text { Domain } \\
\text { affected }\end{array}$ & Predicted effect & Predicted effect on Fas/FasL pathway ${ }^{\complement}$ \\
\hline \multirow[t]{2}{*}{ \#000-220 } & \multirow[t]{2}{*}{$\begin{array}{l}\text { c.[290T > C; } \\
\text { 900_901insA] }\end{array}$} & \multirow[t]{2}{*}{$\begin{array}{l}\text { p.[Phe97Ser; } \\
\text { Ala301Serfs*20] }\end{array}$} & CRD2 & $\begin{array}{l}\text { Possibly damaging } \\
\text { (Score 0.632) }\end{array}$ & \multirow[t]{2}{*}{$\begin{array}{l}\text { Interaction FAS-FASL possibly altered/ } \\
\text { reduced mFAS expression?/Apoptosis } \\
\text { possibly reduced (dominant-negative effect) }\end{array}$} \\
\hline & & & DD & $\begin{array}{l}\text { Possibly damaging } \\
\text { (Score 0.922) }\end{array}$ & \\
\hline \#00800-SD1-1 & c.403_404insT & p.Cys135Leufs*2 & CRD3 & $\begin{array}{l}\text { Probably damaging } \\
\text { (Score 1.000) }\end{array}$ & $\begin{array}{l}\text { Protein expression probably reduced// } \\
\text { abrogated (premature termination before } \\
\text { the TM }>\text { truncated protein) }\end{array}$ \\
\hline \multirow[t]{2}{*}{ \#02M-121 } & $\begin{array}{l}\text { c. [70A > G; } \\
\text { 506_568del] }\end{array}$ & $\begin{array}{l}\text { p.[Asn24Asp; } \\
\text { Gly169_Trp189del] }\end{array}$ & SP & Benign (Score 0.002) & $\begin{array}{l}\text { Isoform FASdeltaExon6 (DTM) }>\text { soluble } \\
\text { protein (sFAS) > reduced mFAS } \\
\text { expression }\end{array}$ \\
\hline & & & TM & - & \\
\hline \#20040730 & c. $418 \mathrm{~T}>\mathrm{C}$ & p.Cys140Arg & CRD3 & $\begin{array}{l}\text { Probably damaging } \\
\text { (Score 1.000) }\end{array}$ & $\begin{array}{l}\text { Interaction FAS-FASL probably altered/ } \\
\text { Reduced mFAS expression? }\end{array}$ \\
\hline \#04011685 & c.403_404insT & p.Cys135Leufs*2 & CRD3 & $\begin{array}{l}\text { Probably damaging } \\
\text { (Score 1.000) }\end{array}$ & $\begin{array}{l}\text { Protein expression probably reduced/ } \\
\text { abrogated (premature termination before } \\
\text { the TM > truncated protein) }\end{array}$ \\
\hline
\end{tabular}

The mutation at the DNA level, the change in the protein, the protein domain affected, the predicted severity of the damage and the predicted effect on the Fas signaling are indicated.

aFrom ATG.

${ }^{b}$ Based on Polyphen-2 software.

Based on literature $21-24$ 
Table 2 Overview of the alterations affecting T-LBL samples

\begin{tabular}{|c|c|c|c|c|c|c|}
\hline Sample code & FAS mutation & FAS expression & FASLG expression & FADD expression & CASP8 expression & CASP3 expression \\
\hline 000-220 & + & $\downarrow$ & nd & nd & nd & nd \\
\hline 00800-SD1-1 & + & $\downarrow$ & nd & nd & nd & nd \\
\hline $02 \mathrm{M}-121$ & + & $\downarrow$ & nd & nd & nd & nd \\
\hline 20040731 & - & $\downarrow$ & - & $\downarrow$ & $\downarrow$ & $\downarrow$ \\
\hline 20050094 & - & $\downarrow$ & - & $\downarrow$ & $\downarrow$ & $\downarrow$ \\
\hline 20040730 & + & $\downarrow$ & - & - & - & - \\
\hline 20041008 & - & $\downarrow$ & - & - & - & - \\
\hline 20040734 & - & $\downarrow$ & - & - & $\downarrow$ & $\downarrow$ \\
\hline 20030516 & - & - & - & $\downarrow$ & $\downarrow$ & $\downarrow$ \\
\hline 20032268 & - & - & - & $\stackrel{\downarrow}{-}$ & $\stackrel{\downarrow}{-}$ & $\stackrel{\downarrow}{-}$ \\
\hline 07010829 & - & - & - & - & - & - \\
\hline 05010346 & - & - & - & - & - & - \\
\hline 01030188 & - & - & - & $\downarrow$ & - & - \\
\hline 02070238 & - & - & - & $\downarrow$ & - & - \\
\hline 02050408 & - & - & - & - & - & - \\
\hline 04011685 & + & $\downarrow$ & - & $\downarrow$ & $\downarrow$ & $\downarrow$ \\
\hline 04010460 & - & 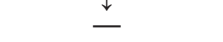 & - & $\stackrel{\downarrow}{-}$ & $\stackrel{\downarrow}{-}$ & $\stackrel{\downarrow}{-}$ \\
\hline 04010153 & - & - & - & - & - & - \\
\hline 11010521 & - & - & - & - & - & - \\
\hline 07010226 & - & - & - & - & - & - \\
\hline 01030192 & - & - & - & - & - & - \\
\hline 04010038 & - & $\downarrow$ & $\uparrow$ & - & - & - \\
\hline 46090749 & - & - & - & - & - & - \\
\hline 04100080 & - & - & - & $\downarrow$ & - & - \\
\hline 04090135 & - & - & - & $\downarrow$ & - & - \\
\hline 04030154 & - & - & - & $\stackrel{\downarrow}{-}$ & - & - \\
\hline Total & $5 / 26(19.2 \%)$ & 10/26 (38.5\%) & $1 / 23(4.3 \%)$ & 8/23 (34.8\%) & $5 / 23(21.7 \%)$ & 5/23 (21.7\%) \\
\hline
\end{tabular}

Summary of the results obtained in our T - LBL sample series, concerning mutations affecting FAS, alterations of FAS expression and alterations of other members of the Fas/FasL pathway. T-LBL cases with any of the elements altered are in bold. The percentage of each alteration type is also indicated. $(+)$, mutation. ( -$)$, normal situation. Arrows denote altered expression. nd, not determined

a
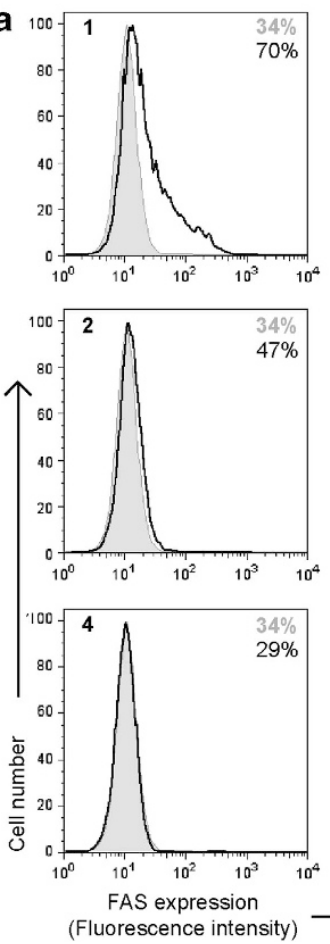

Endogenous

$\square$ Transfected:

1. Wild-type

2. $000-220$

3. $00800-S D 1-1$

4. $02 \mathrm{M}-121$

5. 20040730
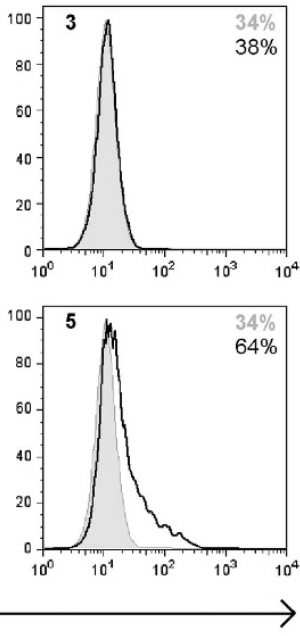

b

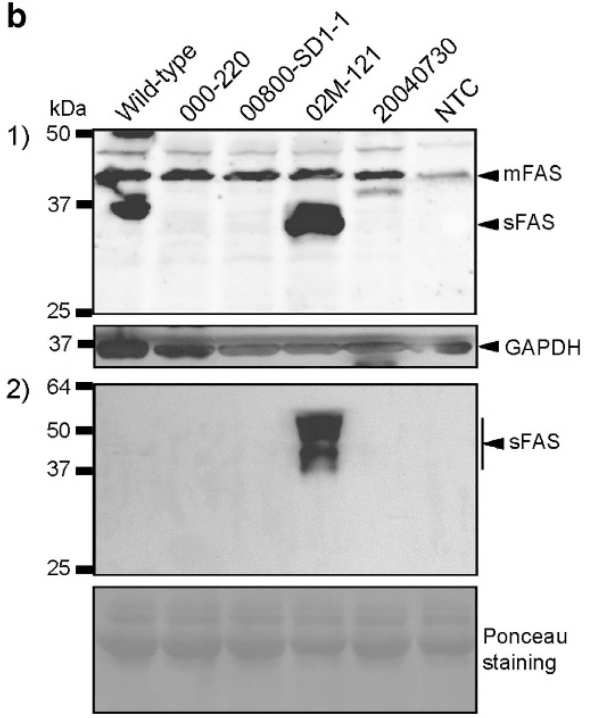

1)

Figure 2 In vitro expression levels of mutant FAS variants. (a) JK cells transfected with wild-type or mutant FAS variant pKEX constructs. Flow cytometry showing endogenous (shaded) and exogenous (solid) surface FAS expression levels $48 \mathrm{~h}$ after transfection. Figure shows a representative of three independent experiments. (b) HEK$293 T$ cells transfected with wild-type or mutant FAS variant pKEX constructs. Western blot of (panel 1) cellular protein extracts and (panel 2) conditioned medium protein extracts. NTC, non-transfected control 


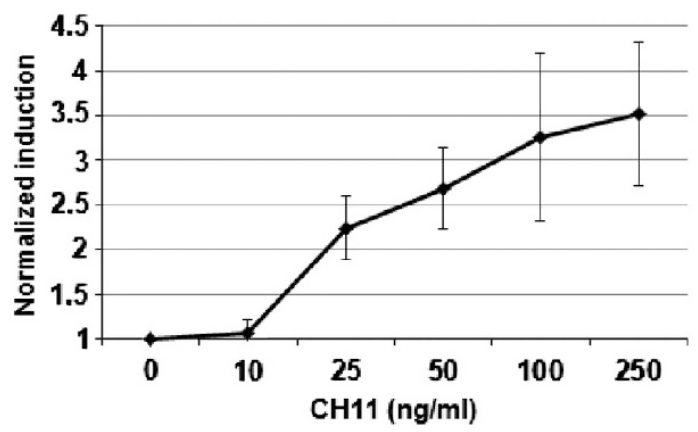

b

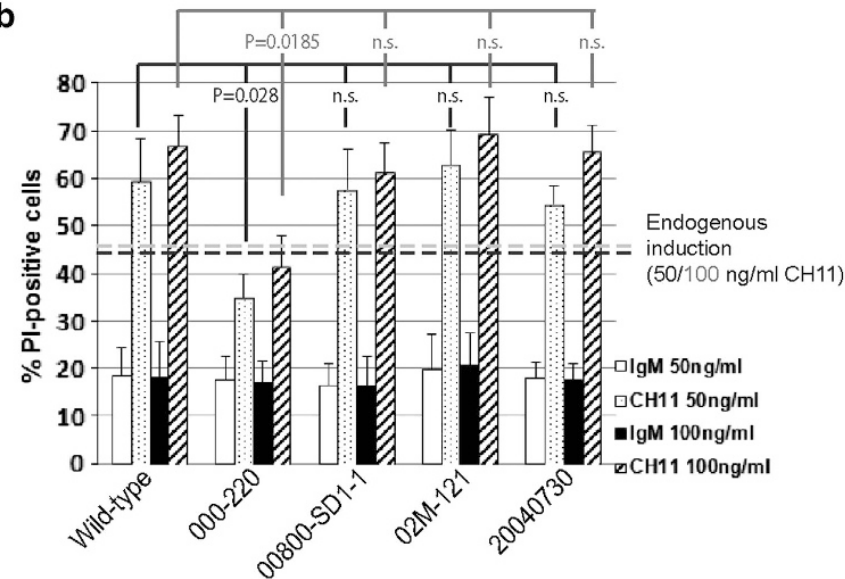

c
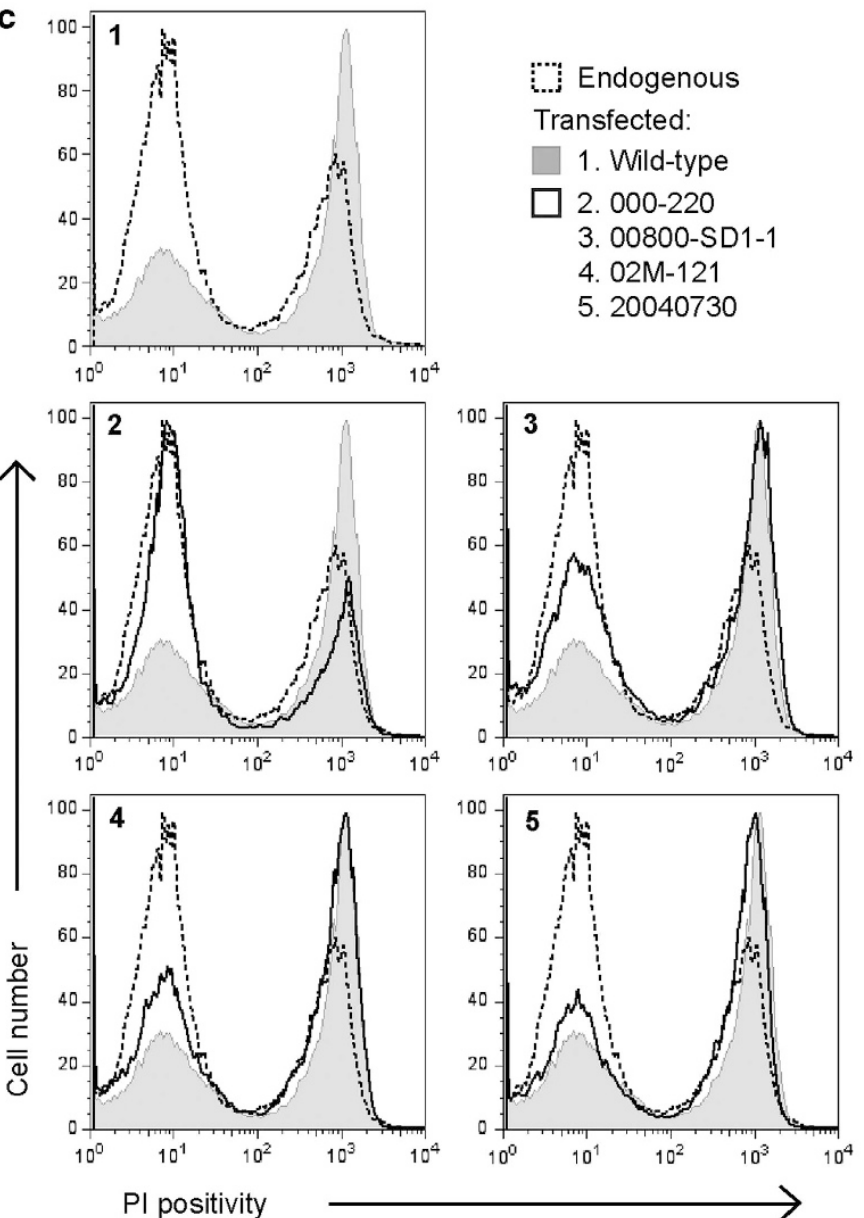

d
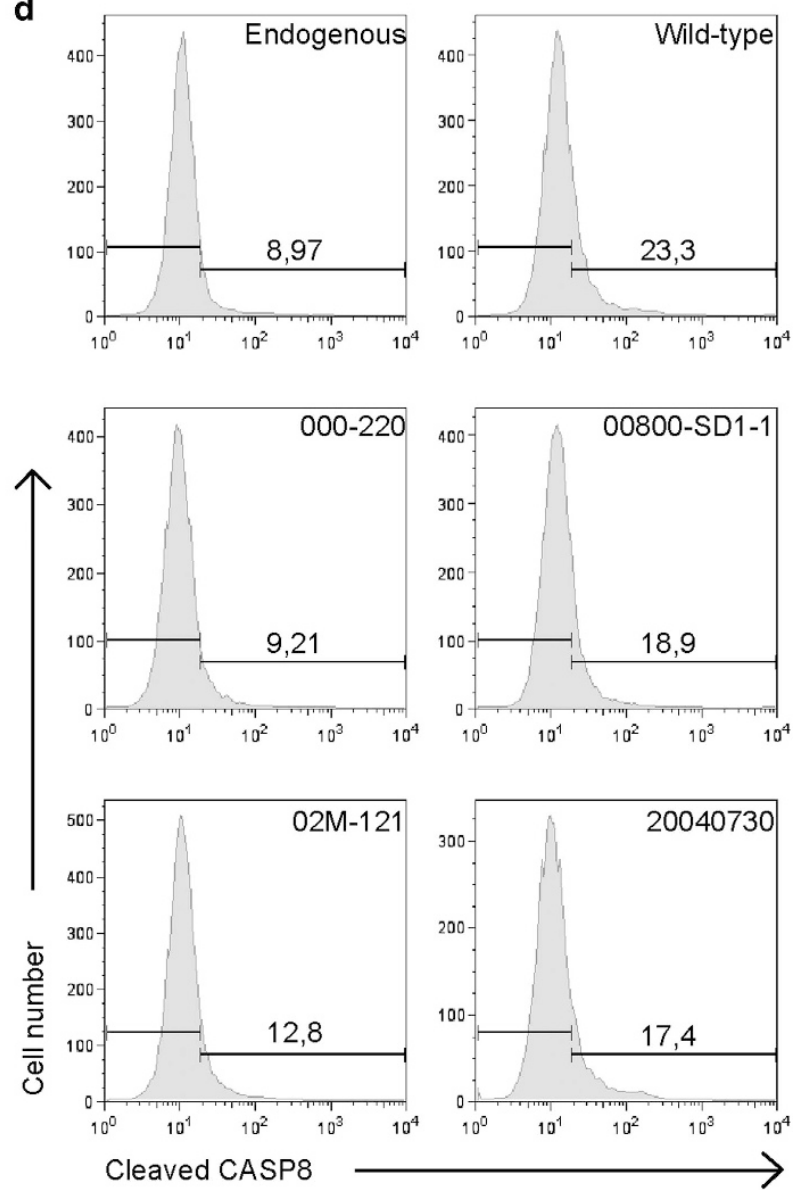

Figure 3 Functionality of mutant FAS variants. (a) Set-up of the effective dose for $\mathrm{CH} 11$-mediated cell death induction, showing the normalized cell death of JK cells treated with increasing doses of $\mathrm{CH} 11$. Normalization was made as follows: first, all data were divided by the result for PI staining of non-stimulated cells $(0 \mathrm{ng} / \mathrm{ml} \mathrm{CH} 11)$, thus setting the latter as 1. Second, the results for PI staining of irrelevant IgM-treated cells were subtracted from their corresponding CH11-treated cells. (b) FAS-mediated cell death induction in JK cells transfected with wild-type or mutant FAS variant pKEX constructs. Histogram represents the mean and S.D. from at least three independent experiments of 50 and $100 \mathrm{ng} / \mathrm{ml}$ of irrelevant IgM and $\mathrm{CH} 11$ induction. The percentage of PI-positive cells is showed, previously subtracting from all data the result for nonstimulated cells. Dotted lines represent the result for \% PI-positive cells treated with $50 \mathrm{ng} / \mathrm{ml}$ (black line) or $100 \mathrm{ng} / \mathrm{ml}$ (gray line) CH11 in JK cells not transfected. Significance is indicated by the corresponding P-value. n.S., not significant. (c) Representative experiment of flow cytometry showing Pl positivity $24 \mathrm{~h}$ after $100 \mathrm{ng} / \mathrm{ml} \mathrm{CH} 11$-triggering by endogenous FAS (dashed line), or exogenous wild-type (shaded) or mutant FAS variants (solid line). (d) FAS-mediated caspase 8 activation in JK cells transfected with wildtype or mutant FAS variants pKEX constructs, $8 \mathrm{~h}$ after $100 \mathrm{ng} / \mathrm{ml} \mathrm{CH} 11$-triggering. Numbers indicate fluorescence intensity corresponding to the cleaved caspase 8 staining 


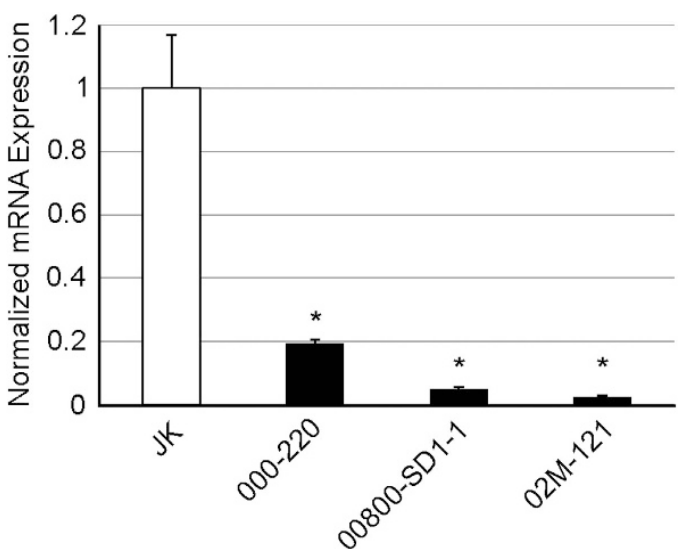

Figure 4 FAS mRNA expression level in RNA samples. qRT-PCR determining FAS mRNA expression levels in human T-LBL samples where only RNA was available. The mean values were normalized to that of JK. Error bars represent S.D. Data represent two independent experiments. *Denotes statistical significance compared with the $\mathrm{JK}$ control, with $P<0.05$

FADD is essential for the apoptotic signal to be transduced inside the cell. A clear reduction of FADD levels was found in 8 out of the 23 samples analyzed (34.8\%), which suggests that loss of FADD may be another frequent mechanism, whereby FAS signaling would be impaired in human T-LBL samples.

CASP8 becomes active by its homotypic interaction with FADD at the DISC complex, which in turn cleaves and activates the effector caspase 3 (CASP3). Clearly reduced CASP8 levels, in its full-length procaspase form, were observed in 5/23 T-LBL samples (21.7\%), indicating that the FAS signaling might as well be blocked at this step of the pathway.

Finally, a clear reduction in procaspase 3 levels were found in the same five T-LBL samples where CASP8 was reduced $(21.7 \%)$, corroborating the blockage of FAS signaling at the most downstream members of the pathway in these samples. Interestingly, both the cleaved intermediate p43/p41 of CASP 8 and the large cleaved fragment p19/p17 of CASP3 were detected in one T-LBL sample (46090749), which indicates the activation of the FAS-signaling pathway in this sample at the precise moment of the biopsy.

In summary, these results suggest that the altered expression of members of the FAS/FASLG pathway other than FAS may as well be involved in the occurrence of T-LBL in humans.

\section{Discussion}

Despite other cellular responses emanating from FAS signaling (reviewed in Ehrenschwender and Wajant ${ }^{11}$ ), its major and best known role is apoptotic cell death. Tumorigenesis may be facilitated by the acquisition of resistance towards FAS-mediated apoptosis through mechanisms involving a variety of processes (reviewed in Villa-Morales and Fernandez-Piqueras ${ }^{22}$ ). Our aim at this study was to determine whether FAS-mediated apoptotic signaling is compromised in human T-LBL samples and the mechanism or mechanisms involved in such alteration.

In a 26-sample series of diagnosed T-LBL human cases, we have found five cases (19.2\%) exhibiting novel heterozygous mutations at FAS. The functional consequences of FAS mutations have been extensively described and they depend on their genetics on the one side, and on the protein domain affected on the other. ${ }^{23-26}$ Homozygous mutations usually provoke a complete impairment of FAS-induced apoptosis in vitro, whereas heterozygous $F A S$ mutations are related to a partial impairment of the apoptotic system functionality. Regarding the domain affected, mutations affecting the ECD may cause reduced mFAS expression; mutations at the CRD1 region of the $E C D$, and thus the PLAD, may result in impaired trimerization of the FAS receptor; mutations at the CRD2-3 regions may result in impaired interaction of FAS receptor with the FAS ligand. This may render the affected cells apoptosis-defective either due to a lack of multimerization of the receptor or the lack of DISC recruitment by the residual FAS molecules. Alterations of the TM domain, like in the case of alternative splicing-deleting exon 6 , will cause reduced mFAS expression in the detriment of sFAS. Mutations affecting the ICD may have no effect on mFAS expression; they can exert however a transdominant-negative effect, since trimerization between wild-type and mutant FAS molecules takes place, but the DISC formation and the subsequent signal transduction is not effective.

In case number 000-220, our results indicate that FAS signaling is impaired (Figure 3), which may be the eventual consequence of FAS mutations in this tumor, probably due to the combination of reduced FAS expression on the surface, impaired FAS-FASLG interaction and defective ability of the DD at signal transduction. This could be even a dominantnegative effect.

Case numbers 00800-SD1-1 and 04011685 exhibited the same point mutation affecting CRD3. Consequent reduction in FAS expression has been demonstrated in these cases through in vitro expression experiments, as well as in the tumor samples (Figures 2a and 4, Table 2), and the suspicion that the protein could be secreted was discarded (Figure $2 b$ ), thus suggesting that this mutant FAS protein may be degraded intracellularly. However, reduced mFAS expression due to the sole presence of such FAS mutation does not account for defective FAS signaling in this case, as shown in Figure 3. Of remarkable interest, however, is the fact that T-LBL case number 04011685 also exhibited a lack of FADD, CASP8 and CASP3 expression, as demonstrated in the biopsies using western blot (Figure 5, Table 2). Altogether, the FAS pathway status of this patient seems to be abrogated at different levels, in what might be the result of cumulative alterations ensuring FAS resistance acquisition.

Case number $02 \mathrm{M}-121$ exhibits a missense mutation at the SP predicted to be benign. Interestingly, it also showed alternative splicing of $F A S$, thus producing the sFAS protein lacking the TM domain. This soluble protein was demonstrated to be secreted to the extracellular medium through in vitro expression experiments (Figure 2b). Moreover, reduced levels of the FAS protein at the cell membrane were found in this case (Figure 2a). The presence of sFAS in the detriment of $\mathrm{mFAS}$ has been implicated in the pathogenesis of autoimmune and malignant diseases, either by antagonizing FASLG or by haploinsufficiency of mFAS expression. ${ }^{25,27,28}$ However, our results indicate that neither mutation-related reduced mFAS expression nor the secretion of sFAS account for defective FAS signaling in this case (Figure 3). 

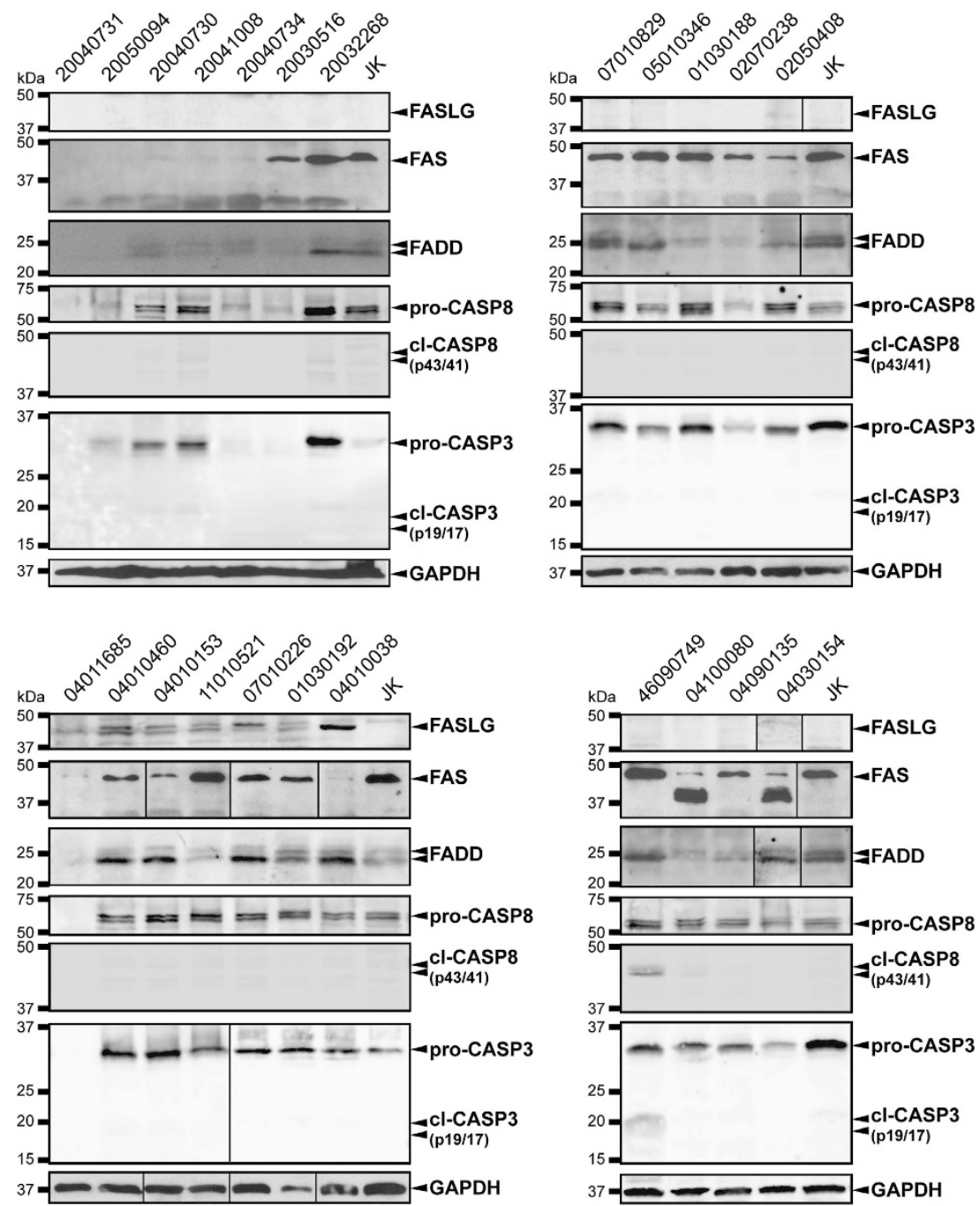

Figure 5 Fas pathway expression levels in human T-LBL samples. Western blot showing FAS, FASLG, FADD, CASP8 and CASP3 protein levels in human T-LBL protein extracts. kDa, kiloDaltons. cl, cleaved

Case number 20040730 exhibits reduced FAS expression in the tumor sample (Figure 5, Table 2). Interestingly, this reduction cannot be solely explained by the occurrence of a mutation, as the in vitro mFAS expression experiments showed a similar level to that of the wild-type sequence (Figure 2). This suggests that regulatory mechanisms might be responsible for the reduction of FAS expression level in the tumor. No defective FAS signaling is detected by our in vitro experiments in this case (Figure 3 ). Considering its normal surface FAS expression, we speculate that the occurrence of the FAS mutation described in this sample does not result in the failure of FAS to bind its ligand either. FADD, CASP8 and CASP3 could be detected in the biopsies using western blot, thus suggesting that the FAS pathway might be only impaired at the FAS expression level in this tumor.

Studies determining the molecular genetics of lymphoblastic neoplasms may however suggest that FAS mutations are not sufficient to compromise FAS function due to their low frequency. In our study, beyond the occurrence of mutations at the FAS gene, 10/26 (38.5\%) human T-LBL samples exhibited a clear reduction in the level of FAS expression, which suggest that reduced FAS expression underlies the occurrence of T-LBL to a wide extent. Reduced expression of FAS has been recurrently reported in the literature as an important mechanism, whereby tumor cells may resist towards FAS-mediated apoptotic signaling. Evidence for FAS downregulation has been shown in bladder cancer, ${ }^{29}$ primary cutaneous $\mathrm{T}$-cell lymphoma, ${ }^{30}$ myeloid leukemia cells, ${ }^{31}$ melanoma, ${ }^{32}$ colorectal carcinoma ${ }^{33}$ or breast cancer. ${ }^{34}$ Moreover, FAS expression levels have been determined in two independent array-based expression profiling studies comparing T-ALL and T-LBL human samples (GEO accession numbers GSE1577 and GSE29986). The results show reduced FAS expression in T-LBL compared with T-ALL, despite these differences not being significant, probably due to the small number of samples analyzed (data not shown).

The percentage of tumor cases showing defective FASmediated apoptotic signaling is usually higher than those bearing FAS mutations or exhibiting reduced FAS expression. 
Thus, other mechanisms involved in FAS signaling must underlie the impairment of those remaining cases. Several tumors can express FASLG, necessarily together with protective loss of FAS expression, this being considered a mechanism by which tumors expressing FASLG escape destruction by the immune response. ${ }^{12,35}$ In our study, FASLG expression, together with loss of FAS, has been detected only in one case (04010038), thus representing a rare event in T-LBL (Table 2). The apoptotic machinery induced by the FAS/FASLG system involves several members whose alteration may as well result in impaired FAS signaling. We found reduced FADD levels in 8 of the 23 samples analyzed (34.8\%; Table 2), suggesting that FADD downregulation may be another frequent mechanism whereby FAS signaling would be impaired in human T-LBL samples. FADD downregulation has been reported in a variety of tumor types, such as non-small cell lung cancer, ${ }^{36}$ hepatocellular carcinoma ${ }^{37}$ or thyroid tumor. ${ }^{38}$ Caspases 8 and 3 may as well be impaired, thus resulting in FAS resistance. In this study, we have found a clear reduction in procaspase 8 and 3 levels in the same five T-LBL samples (Table 2), thus indicating that the pathway might be blocked at the most downstream members of the pathway in roughly $22 \%$ of the T-LBL cases. Taken together, these results suggest that the altered expression of members of the FAS/ FASLG pathway other than FAS may as well be involved in the occurrence of T-LBL in humans.

In conclusion, this study on 26 diagnosed T-LBL samples confirms that the FAS system is impaired to a wide extent in these tumors, with $57.7 \%$ of the cases presenting some sort of alteration of the pathway. A variety of mechanisms seems to be involved in such alteration: in order of frequency, the downregulation of FAS, the deregulation of other members of the pathway and the occurrence of mutations at the FAScoding sequence. Considering these results together, it seems plausible to think of a cumulative effect of several alterations affecting different levels of the FAS/FASLG pathway in each T-LBL, which in turn may result in its deregulation. Since defective FAS signaling may render the T-LBL tumor cells resistant to apoptotic cell death, the correct prognosis, diagnosis and thus the success of anticancer therapy may require such an in-depth knowledge of the complete scenario of FAS-signaling alterations.

\footnotetext{
Materials and Methods

Human samples. Twenty-three human T-LBL samples cryo-protected in optimal cutting temperature-embedding medium were obtained from the Spanish Tumor Bank Network of the Spanish National Cancer Research Centre (Centro Nacional de Investigaciones Oncologicas, CNIO, Madrid, Spain). These samples derive from mass lesions in mediastinum or lymph nodes and are composed of small- to medium-sized blast cells expressing nuclear terminal deoxynucleotidyl transferase. Institutional review board approval was obtained for these studies (references CEI 31-773, UAM Ethics Committee for Research, and RNDdT 10/ 073 ) and the participants provided written informed consent in accordance with the Declaration of Helsinki. RNA from three additional samples was kindly supplied by J. Benítez and B. Martínez. Additional information is summarized in Supplementary Table S1.
}

Cell culture. HEK-293T and JK cell lines were purchased from the American Type Culture Collection (ATCC, Manassas, VA, USA). Human HEK-293T cells were cultured in Dulbecco's modified Eagle's medium (DMEM; Gibco, Life Technologies, Carlsbad, CA, USA) with 10\% FBS (PAA Laboratories, GE
Healthcare Life Sciences, Velizy-Villacoublay, France), $2 \mathrm{mM} \mathrm{L-Glutamine} \mathrm{(Merck}$ KGaA, Darmstadt, Germany) and sodium pyruvate ( $1 \mathrm{mM}$; Merck). Human JK T cells were cultured in RPMl 1640 (Gibco) supplemented with 10\% FBS (PAA Laboratories), L-glutamine ( $2 \mathrm{mM}$; Merck) and $1 \times$ Minimum Essential Medium (MEM) nonessential amino acids.

Cultures were maintained at $37{ }^{\circ} \mathrm{C}$ in a $5 \% \mathrm{CO}_{2}$ humidified atmosphere.

Macromolecule extraction from human samples. Total RNA, DNA and proteins from T-LBL human samples were extracted using TriPure Reagent (Roche Applied Science, Indianapolis, IN, USA), following manufacturer's instructions. RNA extraction method was followed by $\mathrm{AcNH}_{4}-\mathrm{EtOH}$ precipitation.

FAS cDNA amplification and sequencing. Reverse transcription was performed using SuperScript First-Strand Synthesis System for RT-PCR (Invitrogen, Life Technologies) followed by PCR. For PCR, the Fast Start High Fidelity PCR System (Roche Applied Science) was used, with an optimal concentration of dimethyl sulfoxide (DMSO) of $6 \%$. Conditions for PCR were as indicated by the manufacturer, with an optimal annealing temperature of $57.4^{\circ} \mathrm{C}$. Forward primer incorporated Smal recognition site in $5^{\prime}$ (underlined). Primer sequences were as follows:

Smal-Forward: 5'-CCCGGGTTGGTGGACCCGCTCAGTAC-3'

Reverse: $5^{\prime}$-CCCAGTAAAAAACCAAGCAG-3'

PCR products were purified using Wizard SV Gel and PCR Clean-up System (Promega Corporation, Madison, WI, USA) and cloned into pGEM-T-Easy vector system (Promega). Sequencing reactions of several clones from each product were performed using an ABI Prism 310 Automated Sequencer or a 3730XL Sequencer (Applied Biosystems, Life Technologies). All comparisons between sequences were made using the program L-Align from ExPASy Molecular Biology Server.

Cloning of the FAS cDNA sequences. Purified DNA fragments containing the FAS cDNA were cloned into pKEX-FAS expression plasmid (kindly gifted by Peter Krammer, Deutsches Krebsforschungszentrum, Heidelberg, Germany) when necessary, through consecutive digestion with Smal and Xbal (Roche Applied Science).

Transient transfections. HEK-293T cells were transfected using LipofectAMINE Reagent (Invitrogen), following manufacturer's instructions. JK cells were transfected by electroporation using a Gene Pulser MXcell Electroporation System (Bio-Rad Laboratories, Hercules, CA, USA). A total of $10^{7} \mathrm{JK}$ cells in $500 \mu \mathrm{l}$ of complete medium were subjected to $280 \mathrm{~V}, 950 \mu \mathrm{F}$, with $20 \mu \mathrm{g}$ of DNA. Electroporated cells were recovered in $10 \mathrm{ml}$ of complete medium and analysed 48-h post transfection.

Real-time quantitative RT-PCR. The determination of the FAS transcript levels was performed by quantification assays based on real-time qRT-PCR with a LightCycler instrument (Roche Diagnostics $\mathrm{GmbH}$, Mannheim, Germany), using LightCycler RNA Master SYBR Green I (Roche Applied Science) and following manufacturer's instructions. Optimal annealing temperature was $52^{\circ} \mathrm{C}$. The gene encoding the $\beta 2$-microglobulin ( $\beta 2 \mathrm{MG}$ ) was used as an internal control of the RNA quality and amplification. Primers were as follows:

FAS-Fw: $5^{\prime}$-TCTTTCACTTCGGAGGATTG-3'

FAS-Rv: 5'-GCCTTCCAAGTTCTGAGTCT-3'

B2MG-Fw: 5'-CCAGCAGAGAATGGAAAGTC-3'

B2MG-Rv: 5'-GATGCTGCTTACATGTCTCG-3'

All PCRs were repeated for each sample in two independent experiments. FAS expression levels were calculated with the LightCycler Relative Quantification Software (Roche Diagnostics $\mathrm{GmbH}$ ).

Western blot. HEK-293T cells transfected with wild-type and tumor-derived FAS pKEX constructs were assayed for exogenous FAS expression, in order to see both the proper functionality of the constructs and the possible variations in the expression levels among samples. Twenty-four hours after transfection, cells were allowed for conditioning medium (without FBS) for $24 \mathrm{~h}$. Then, proteins from the conditioned medium were extracted based on acetone precipitation. Total proteins from the cells were also obtained, using lysis buffer containing $50 \mathrm{mM}$ Tris- $\mathrm{HCl}, \mathrm{pH}$ 7.4; $1 \% \mathrm{NP}-40 ; 0.25 \%$ sodium deoxycholate; $150 \mathrm{mM} \mathrm{NaCl} ; 1 \mathrm{mM}$ EGTA; $1 \mathrm{mM} \mathrm{PMSF} ; 1 \mathrm{mM} \mathrm{Na} \mathrm{VO}_{4}$; and $2.5 \mu \mathrm{l} / \mathrm{ml}$ of Protease Inhibitor Cocktail (Sigma-Aldrich, St. Louis, MO, USA). Thirty micrograms of cellular protein and $15 \mu \mathrm{l}$ of conditioned medium protein were electrophoresed in 10\% SDS-PAGE 
without and with $\beta$-mercaptoethanol, respectively, and electrotransferred to Immobilon-P transfer membranes (Merck Millipore, Merck).

Thirty microgram aliquots of total protein from human T-LBL samples were separated by $10 \%$ SDS-PAGE under reducing conditions and electrotransferred to Immobilon-P transfer membranes (Merck Millipore, Merck).

Antibodies used were as follows: anti-FAS antibody (clone CH11) (UpstateMerck Millipore, Lake Placid, NY, USA); anti-FADD polyclonal antibody (Santa Cruz Biotechnology Inc., Dallas, TX, USA); anti-FASLG polyclonal antibody, anti-CASP8 antibody (clone 1C12), anti-cleaved CASP8 (D391) antibody (clone 18C8), antiCASP3 polyclonal antibody (all from Cell Signaling Technology Inc., Danvers, MA, USA); anti-GAPDH antibody (clone 1D4; Enzo Life Sciences Inc., Farmingdale, NY, USA); and the corresponding secondary antibodies: anti-mouse IgM, HRPconjugated (Nordic Immunology, Tilburg, The Netherlands); anti-mouse IgG, HRP. conjugated, anti-rabbit lgG, HRP-conjugated (Cell Signaling). More information is summarized in Supplementary Table S2.

The peroxidase activity was developed using WesternBright ECL Detection System (Advansta Inc., Menlo Park, CA, USA).

ImageQuant LAS 4000 digital imaging system (GE Healthcare Bio-Sciences Corp., Piscataway, NJ, USA) was used for acquisition of images.

Flow cytometry. Surface FAS expression was determined in $10^{5}$ cells preincubated with FITC labeled anti-FAS antibody, clone UB2 (MBL International, Woburn, MA, USA) or FITC Mouse IgG1 $\kappa$ Isotype Control (BD Pharmingen, BD Biosciences, San Jose, CA, USA) for $30 \mathrm{~min}$ at room temperature. The cells were then washed with $1 \times$ PBS containing $1 \%$ bovine serum albumin $(B S A)$, $1 \times$ heat-inactivated FBS and $0.01 \%$ sodium azide, and analyzed by flow cytometry on a FACSCalibur (Becton-Dickinson, Franklin Lakes, NJ, USA) for percentage and mean fluorescence intensity.

Cleaved caspase 8 was determined by incubating cells with anti-cleaved CASP8 (D391) antibody (clone 18C8) (Cell Signaling) for $30 \mathrm{~min}$ at room temperature, then with Alexa Fluor 488 Donkey Anti-Rabbit lgG $(\mathrm{H}+\mathrm{L}$ ) Antibody (Molecular Probes, Life Technologies, Carlsbad, CA, USA). Cells were then washed with $1 \times$ PBS containing $0.5 \% \mathrm{BSA}$ and analyzed by flow cytometry on a FACSCalibur (Becton-Dickinson) for percentage and mean fluorescence intensity.

FAS signaling induction. Twenty-four hours after transfection (where indicated), $2 \times 10^{5} \mathrm{JK}$ cells at $10^{6} \mathrm{cells} / \mathrm{ml}$ in 96 -well plates were treated for $24 \mathrm{~h}$ (for PI staining) or $8 \mathrm{~h}$ (for cleaved caspase 8 staining) with anti-FAS mouse monoclonal antibody (clone $\mathrm{CH} 11$; Upstate-Merck Millipore) or mouse $\operatorname{lgM} \lambda$ isotype control (clone 11E10; Beckman Coulter, Nyon, Switzerland). Then the cells were either incubated with $2 \mu \mathrm{g} / \mathrm{ml} \mathrm{PI} \mathrm{(Sigma-Aldrich)} \mathrm{and} \mathrm{tested}$ for cell death ( $\mathrm{Pl}$ positivity), or fixed and permeabilized using $4 \%$ paraformaldehyde (Panreac Química S.L.U., Barcelona, Spain) and 90\% methanol (Merck) for cleaved caspase 8 staining, using in both cases a FACSCalibur (Becton-Dickinson)

Software. Polyphen-2 (http://genetics.bwh.harvard.edu/pph2/) and SIFT Human Protein (http://sift.jcvi.org/www/SIFT enst submit.html) softwares were used to predict the effect of mutations on the functionality of the protein.

The FlowJo software was used for analysis after flow cytometry.

Statistical analyses. Statistical significances were determined by a Student's $t$-test, using the Statistical Package for the Social Sciences software (SPSS version 15.0, IBM Corporation, Somers, NY, USA).

\section{Conflict of Interest}

The authors declare no conflict of interest.

1. Cortelazzo S, Ponzoni M, Ferreri AJ, Hoelzer D. Lymphoblastic lymphoma. Crit Rev Oncol Hematol 2011; 79: 330-343.

2. Bonn BR, Rohde M, Zimmermann M, Krieger D, Oschlies I, Niggli F et al. Incidence and prognostic relevance of genetic variations in T-cell lymphoblastic lymphoma in childhood and adolescence. Blood 2013; 121: 3153-3160.

3. de Leval L, Bisig B, Thielen C, Boniver J, Gaulard P. Molecular classification of T-cell lymphomas. Crit Rev Oncol Hematol 2009; 72: 125-143.

4. Oehm A, Behrmann I, Falk W, Pawlita M, Maier G, Klas C et al. Purification and molecular cloning of the APO-1 cell surface antigen, a member of the tumor necrosis factor/nerve growth factor receptor superfamily. Sequence identity with the Fas antigen. J Biol Chem 1992; 267: 10709-10715.
5. Suda T, Takahashi T, Golstein P, Nagata S. Molecular cloning and expression of the Fas ligand, a novel member of the tumor necrosis factor family. Cell 1993; 75 : 1169-1178.

6. Papoff G, Hausler P, Eramo A, Pagano MG, Di Leve G, Signore A et al. Identification and characterization of a ligand-independent oligomerization domain in the extracellular region of the CD95 death receptor. J Biol Chem 1999; 274: 38241-38250.

7. Takahashi T, Tanaka M, Brannan Cl, Jenkins NA, Copeland NG, Suda T et al. Generalized lymphoproliferative disease in mice, caused by a point mutation in the Fas ligand. Cell 1994; 76: 969-976.

8. Muzio M, Chinnaiyan AM, Kischkel FC, O'Rourke K, Shevchenko A, Ni J et al. FLICE, a novel FADD-homologous ICE/CED-3-like protease, is recruited to the CD95 (Fas/APO-1) death-inducing signaling complex. Cell 1996; 85: 817-827.

9. Medema JP, Scaffidi C, Kischkel FC, Shevchenko A, Mann M, Krammer PH et al. FLICE is activated by association with the CD95 death-inducing signaling complex (DISC). EMBO J 1997; 16: 2794-2804

10. Baumann S, Krueger A, Kirchhoff S, Krammer PH. Regulation of $\mathrm{T}$ cell apoptosis during the immune response. Curr Mol Med 2002; 2: 257-272.

11. Ehrenschwender M, Wajant $\mathrm{H}$. The role of FasL and Fas in health and disease. Adv Exp Med Biol 2009; 647: 64-93.

12. Muschen M, Warskulat U, Beckmann MW. Defining CD95 as a tumor suppressor gene. J Mol Med (Berl) 2000; 78: 312-325.

13. Villa-Morales M, Gonzalez-Gugel E, Shahbazi MN, Santos J, Fernandez-Piqueras J. Modulation of the Fas-apoptosis-signalling pathway by functional polymorphisms at Fas, FasL and Fadd and their implication in T-cell lymphoblastic lymphoma susceptibility. Carcinogenesis 2010; 31: 2165-2171.

14. Villa-Morales M, Santos J, Fernandez-Piqueras J. Functional Fas (Cd95/Apo-1) promoter polymorphisms in inbred mouse strains exhibiting different susceptibility to gamma-radiation-induced thymic lymphoma. Oncogene 2006; 25: 2022-2029.

15. Villa-Morales M, Santos J, Perez-Gomez E, Quintanilla M, Fernandez-Piqueras J. A role for the Fas/FasL system in modulating genetic susceptibility to T-cell lymphoblastic lymphomas. Cancer Res 2007; 67: 5107-5116.

16. Tauzin S, Debure L, Moreau JF, Legembre P. CD95-mediated cell signaling in cancer: mutations and post-translational modulations. Cell Mol Life Sci 2012; 69: 1261-1277.

17. Beltinger C, Kurz E, Bohler T, Schrappe M, Ludwig WD, Debatin KM. CD95 (APO-1/Fas) mutations in childhood T-lineage acute lymphoblastic leukemia. Blood 1998; 91 : 3943-3951.

18. Rozenfeld-Granot G, Toren A, Amariglio N, Brok-Simoni F, Rechavi G. Mutation analysis of the FAS and TNFR apoptotic cascade genes in hematological malignancies. Exp Hematol 2001; 29: 228-233.

19. den Dunnen JT, Antonarakis SE. Mutation nomenclature extensions and suggestions to describe complex mutations: a discussion. Hum Mutat 2000; 15: 7-12.

20. Altznauer F, von Gunten S, Spath P, Simon HU. Concurrent presence of agonistic and antagonistic anti-CD95 autoantibodies in intravenous Ig preparations. J Allergy Clin Immunol 2003; 112: 1185-1190.

21. Chodorge M, Zuger S, Stirnimann C, Briand C, Jermutus L, Grutter MG et al. A series of Fas receptor agonist antibodies that demonstrate an inverse correlation between affinity and potency. Cell Death Differ 2012; 19: 1187-1195.

22. Villa-Morales M, Fernandez-Piqueras J. Targeting the Fas/FasL signaling pathway in cancer therapy. Expert Opin Ther Targets 2012; 16: 85-101.

23. Fisher GH, Rosenberg FJ, Straus SE, Dale JK, Middleton LA, Lin AY et al. Dominant interfering Fas gene mutations impair apoptosis in a human autoimmune lymphoproliferative syndrome. Cell 1995; 81: 935-946.

24. Magerus-Chatinet A, Neven B, Stolzenberg MC, Daussy C, Arkwright PD, Lanzarotti N et al. Onset of autoimmune lymphoproliferative syndrome (ALPS) in humans as a consequence of genetic defect accumulation. J Clin Invest 2011; 121: 106-112.

25. Roesler J, Izquierdo JM, Ryser M, Rosen-Wolff A, Gahr M, Valcarcel J et al. Haploinsufficiency, rather than the effect of an excessive production of soluble CD95 (CD95\{Delta\}TM), is the basis for ALPS la in a family with duplicated 3' splice site AG in CD95 intron 5 on one allele. Blood 2005; 106: 1652-1659.

26. Vaishnaw AK, Orlinick JR, Chu JL, Krammer PH, Chao MV, Elkon KB. The molecular basis for apoptotic defects in patients with CD95 (Fas/Apo-1) mutations. J Clin Invest 1999; 103: 355-363.

27. Cascino I, Fiucci G, Papoff G, Ruberti G. Three functional soluble forms of the human apoptosis-inducing Fas molecule are produced by alternative splicing. J Immunol 1995; 154: 2706-2713.

28. Cheng J, Zhou T, Liu C, Shapiro JP, Brauer MJ, Kiefer MC et al. Protection from Fas-mediated apoptosis by a soluble form of the Fas molecule. Science 1994; 263: 1759-1762.

29. Watson CJ, O'Kane H, Maxwell P, Sharaf O, Petak I, Hyland PL et al. Identification of a methylation hotspot in the death receptor Fas/CD95 in bladder cancer. Int J Oncol 2012; 40: 645-654.

30. Jones CL, Wain EM, Chu CC, Tosi I, Foster R, McKenzie RC et al. Downregulation of Fas gene expression in Sezary syndrome is associated with promoter hypermethylation. $J$ Invest Dermatol 2010; 130: 1116-1125.

31. Blomberg J, Ruuth K, Jacobsson M, Hoglund A, Nilsson JA, Lundgren E. Reduced FAS transcription in clones of U937 cells that have acquired resistance to Fas-induced apoptosis. FEBS J 2009; 276: 497-508. 
32. Bullani RR, Wehrli P, Viard-Leveugle I, Rimoldi D, Cerottini JC, Saurat JH et al. Frequent downregulation of Fas (CD95) expression and function in melanoma. Melanoma Res 2002 12: 263-270.

33. Wang WS, Chen PM, Wang HS, Liang WY, Su Y. Matrix metalloproteinase-7 increases resistance to Fas-mediated apoptosis and is a poor prognostic factor of patients with colorectal carcinoma. Carcinogenesis 2006; 27: 1113-1120.

34. Mottolese M, Buglioni S, Bracalenti C, Cardarelli MA, Ciabocco L, Giannarelli D et al. Prognostic relevance of altered Fas (CD95)-system in human breast cancer. Int $\mathrm{J}$ Cancer 2000; 89: 127-132.

35. Kennedy-Nasser AA, Hanley P, Bollard CM. Hodgkin disease and the role of the immune system. Pediatr Hematol Oncol 2011; 28: 176-186.

36. Cimino $\mathrm{Y}$, Costes A, Damotte D, Validire P, Mistou S, Cagnard N et al. FADD protein release mirrors the development and aggressiveness of human non-small cell lung cancer. Br J Cancer 2012; 106: 1989-1996.
37. Schattenberg JM, Schuchmann M, Galle PR. Cell death and hepatocarcinogenesis: Dysregulation of apoptosis signaling pathways. J Gastroenterol Hepatol 2011; 26(Suppl 1): 213-219.

38. Tourneur L, Mistou S, Michiels FM, Devauchelle V, Renia L, Feunteun J et al. Loss of FADD protein expression results in a biased Fas-signaling pathway and correlates with the development of tumoral status in thyroid follicular cells. Oncogene 2003; 22: 2795-2804.

(i) $\odot$ Cell Death and Disease is an open-access journal published by Nature Publishing Group. This work is licensed under a Creative Commons Attribution-NonCommercialNoDerivs 3.0 Unported License. To view a copy of this license, visit http://creativecommons.org/licenses/by-nc-nd/3.0/

Supplementary Information accompanies this paper on Cell Death and Disease website (http://www.nature.com/cddis) 